\title{
The effect of different dietary structure on gastrointestinal dysfunction in children with cerebral palsy and epilepsy based on intestinal flora
}

\section{Anquan Zhang ( $\triangle 928572424 @ q q . c o m$ )}

Fuyong People's hospital https://orcid.org/0000-0003-1565-2469

\section{Congfu Huang}

Longgang District Maternity\&Child Healthcare Hospital,Shenzhen

\section{Xiuyun Li}

Longgang District Maternity\&Child Healthcare Hospital,Shenzhen

\section{Yuanping Peng}

Longgang District Social Welfare Center,Shenzhen

\section{Zhenyu Yang}

School of Statistic and Data Science, Nankai University

\section{Lan Ge}

BGI Nutrition Precision.,Ltd,Shenzhen

\section{Genfeng Wu}

Longgang District Maternity\&Child Healthcare Hospital,Shenzhen

\section{Shuyuan Huang}

Longgang District Social Welfare Center,Shenzhen

\section{Peiqin Wang}

Longgang District Maternity\&Child Healthcare Hospital,Shenzhen

\section{Wenkui Dai}

Department of Microbial Research,WeHealthGene Institute,Shenzhen

\section{Yansi Lyu}

Shenzhen University General Hospital,Shenzhen

\section{Linlin Wang}

Shenzhen University General Hospital,Shenzhen

\section{Research article}

Keywords: Obstipation, Cerebral palsy, Epilepsy, Intestinal flora, Gastrointestinal dysfunction

Posted Date: January 16th, 2020

DOl: https://doi.org/10.21203/rs.2.20994/v1 
License: (c) (i) This work is licensed under a Creative Commons Attribution 4.0 International License. Read Full License 
The effect of different dietary structure on gastrointestinal dysfunction in children with cerebral palsy and epilepsy based on intestinal flora

Huang Congfu ${ }^{1, *}$, Li Xiuyun ${ }^{1}$, Peng Yuanping ${ }^{4}$, Yang zhenyu ${ }^{2}$, Ge Lan $^{3}$, Wu GenFeng ${ }^{1}$, Huang Shuyuan $^{4}$, Wang Peiqin ${ }^{1}$, Dai Wenkui ${ }^{2}$, Lyu yansi ${ }^{5}$, Wang Linlin ${ }^{5}$, Zhang Anquan ${ }^{6}$

1. *Longgang District Maternity \& Child Healthcare Hospital, Shenzhen, China, 518172

2. Yang zhenyu: School of Statistics and Data Science, Nankai University, Tianjin, China, 300000 ;

Dai Wenkui: Department of Microbial Research, WeHealthGene Institute, Shenzhen, Guangdong, China, 518000; Joint Laboratory of Micro-ecology and Children's Health, Shenzhen Children's Hospital \& Shenzhen WeHealthGene Co. Ltd., Shenzhen, Guangdong, China,518000

3. BGI Nutrition Precision Co., Ltd, Shenzhen, China, 518083

4. Longgang District Social Welfare Center, Shenzhen, China, 518116

5. Shenzhen University General Hospital, Shenzhen, China, 518000

6. Bao'an District Fuyong People's Hospital, Shenzhen, China, 510370

Corresponding author: Zhang Anquan, E-mail: 928572424@qq.com

Name of program: Longgang District Science and Technology Innovation Bureau (no. p020180720388406844161)

Primary author: Huang Congfu (1975-), associate chief physician on nerve and respiratory system diseases of children,78333755@qq.com

Correspondence to: Zhang Anquan (1976-), associate chief physician on nerve and respiratory system diseases of children, 928572424@qq.com 


\begin{abstract}
Background: Gastrointestinal (GI) difficulties are very common among children with cerebral palsy (CP) and comorbid epilepsy. GI function is influenced by dietary structure on intestinal flora. The aim of this study was to compare intestinal flora changes in two dietary groups of this population and examine whether such differences are related to GI dysfunction.
\end{abstract}

Methods: Forty children with CP and epilepsy were recruited from among patients being cared for at a social welfare center, including 23 consuming a (semi)fluid diet (liquid diet group) and 17 consuming a normal diet (general diet group). Bacterial DNA was extracted from feces, the V3-V4 region of the 16S rRNA gene was amplified from the DNA, and high-throughput sequencing of the amplified sequences was performed. Microbe prevalence levels were compared on multiple phylogenic levels.

Results: Gut microbial populations differed substantially between the liquid diet and general diet groups. The only two phyla that differed significantly between the two groups were Bacteroidetes $(p=$ 0.034) and Actinobacteria $(p=0.013)$. Regarding representation of genera, Prevotella species were selectively predominant in the general diet group $(25.849 \%$ vs. $3.612 \%$ in the liquid diet group, $p<$ 0.001), while Bifidobacterium species were selectively predominant in the liquid diet group (24.929\% vs. $12.947 \%$ in the general diet group, $p=0.013$ ). The gut flora of children in the general diet group contained more butyric acid-producing flora which was also common in healthy people (e.g. Lachnoclostridium, Dorea, Ruminococcus, Faecalibacterium, Roseburia, and Coprococcus). The gut flora of children in liquid diet group however, were rich in symbiotic pathogenic bacteria (e.g. Collinsella, Alistipes, and Eggerthella).

Conclusion: The intestinal flora of children with CP and epilepsy consuming a liquid diet had elevated levels of symbiotic pathogens and diminished intestinal barrier protection bacteria, relative to a general diet group. These alterations in bacterial flora were associated with GI dysfunction symptoms. 
Key words Obstipation; Cerebral palsy; Epilepsy; Intestinal flora; Gastrointestinal dysfunction

\section{Background}

Commonly, children with cerebral palsy (CP) experience multiple central nervous system dysfunctions. Epilepsy has been reported to be present in some $43 \%$ of pediatric CP patients (range, 35-62\%) [1]. Quitadamo reported that nearly nine in ten children with $\mathrm{CP}$ experience digestive system problems - such as constipation, difficulty eating, salivation problems, dysphagia, gastroesophageal reflux, and among them constipation would affect $26 \%-74 \%$ children with cerebral palsy [2]. In our clinical experience, we find that almost every child with CP and comorbid epilepsy has some degree of gastrointestinal (GI) dysfunction, with the most bothersome one reported being obstipation, followed by bloating, vomiting, and recurrent GI bleeding [3].

GI function can be modulated by dietary influences on intestinal flora $[4,5,6,7,8]$. The present study included a group of children diagnosed with CP and epilepsy who ate a fluid or semifluid diet and a similarly diagnosed reference group of children consuming a normal diet. We compared GI microbial diversity between the two groups, and examined whether the children's GI difficulties could be related to their intestinal flora characteristics.

\section{Methods}

\section{Patients and Study Design}

A cohort of 40 children being cared for at the Longgang District Social Welfare Center who had CP and epilepsy diagnoses were enrolled in this study, including 23 on a (semi)fluid diet (liquid diet group) and 17 who were consuming a normal diet (general diet group). (Semi) fluid diets consisted predominantly of powdered milk as well as rice (paste) and soup. Normal diets included primarily 
plant-based solid foods (i.e. cereals, potatoes, beans, fruits, vegetables, and products derived from these ingredients), as well as modest amounts of animal proteins and fats. The CP and epilepsy diagnoses were made by clinicians in the neurology departments of the three major hospitals in the region. Patients who had been diagnosed with a metabolic disease or a severe infection and those taking antibiotics or probiotics within 2 weeks before sample collection were not eligible for inclusion in the study. Informed consent was obtained from the legal guardian of each enrolled child.

\section{Fecal sample collection and processing}

Fecal specimens were collected from all participating patients; $\sim 5 \mathrm{~g}$ was taken from the middle and center of each sample as a study specimen. Each 5 -g specimen was frozen after collection at $-80{ }^{\circ} \mathrm{C}$ in 30 min, and transported in dry ice to Beijing Nuohe Zhiyuan Technology Co., Ltd. for high-throughput sequencing. Microbial whole DNA was extracted from the specimens with MoBio's PowerSoil ${ }^{\circledR}$ DNA Isolation Kit, and then the V3-V4 region of the $16 \mathrm{~S}$ rRNA gene was amplified by polymerase chain reaction. Universal primers (338F and $806 \mathrm{R})$ were used with following condition: initial denaturation at $95^{\circ} \mathrm{C}$ for $3 \mathrm{~min}$, followed by 27 cycles of denaturation at $95^{\circ} \mathrm{C}$ for $30 \mathrm{sec}$, annealing at $55^{\circ} \mathrm{C}$ for 30 sec, and extension at $72^{\circ} \mathrm{C}$ for $30 \mathrm{sec}$. The amplified sequence was subjected to high throughput sequencing in the Illumina Miseq Platform.

\section{Analysis of sequencing data and species annotation}

The sequencing data were filtered by a bioinformatics tool developed in our laboratory (available upon request), and the sequences were spliced with Flash software (v1.2.11, http://ccb.jhu.edu/software/flash/index.shtml). The spliced sequences were aggregated in USEARCH into operational taxonomic units, which were compared and annotated based on a bacterial library (Greengene v201305) to yield bacterial composition data for each specimen. The bacterial abundances 
of the specimens were analyzed on the level of phylum, class, order, family, and genus.

\section{Statistical analysis}

Statistics were conducted in R software (v3.3.3) with the ade4 software package. Principle component analysis (PCA) was performed based on the composition and relative abundance of genera and the overall flora distributions of samples by group were plotted. Wilcoxon rank-sum test was used to detect inter-group differences in the presence of phyla and genera. The Benjamini-Hochberg method was applied to adjust the difference analysis results. Means are reported with standard deviations (SDs). $\mathrm{P}$ values $<0.05$ and false discovery rates $(\mathrm{FDRs})<0.05$ were considered statistically significant.

\section{Results}

\section{GI symptoms}

The incidence of GI dysfunction in the liquid diet group was significantly higher than that in the general diet group $(p<0.05)$. With the exception of diarrhea, each examined symptom was found significantly more frequently in the former group than in the latter (Table 1).

\section{Phylum analysis of dominant bacteria and microbial composition}

The percentages of the top-five most abundant bacterial phyla found in each group are reported in Table 2. A histogram of the relative abundance levels of bacterial phyla, by group, based on bacterial structure analysis is shown in Figure 1. The five most abundant bacterial phyla found in the general diet group were Bacteroidetes, Firmicutes, Actinobacteria, Proteobacteria, and Fusobacteria. The five most abundant bacterial phyla found in the liquid diet group were Firmicutes, Actinobacteria, 
Bacteroidetes, Proteobacteria, and Verrucomicrobia. Only the Bacteroides $(p=0.034)$ and Actinobacteria $(p=0.013)$ phyla differed significantly between the two groups.

\section{Genus analysis of dominant bacteria and microbial composition}

The proportions of the top 15 flora, by abundance, were determined for each group and compared, as reported in Table 3. Two of the top-three genera for the general diet group (Prevotella, Bifidobacteria, and Bacteroides) were also in the top-three genera for the liquid diet group (Bifidobacteria, Bacteroides, and Enterococcus). There were numerous genera whose representations differed significantly between the two groups, the 15 most significant of which are reported in Table 4. Notably, Prevotella, Roseburia, Latobacillus, and Faecalibacterium were markedly more abundant in the general diet group than in the liquid diet group, whereas Bifidobacterium and Collinsella were markedly more abundant in the liquid diet group than in the general diet group.

Further analysis of gut microbiota exhibiting relative abundance levels greater than $0.1 \%$ together with significant inter-group differences at the genus level showed a pronounced enrichment of Prevotella in the general diet group (25.85\%) relative to levels in the liquid diet group (3.61\%), as well as a near two-fold enrichment of Bifidobacterium representation in liquid diet group (24.93\%) compared with levels in the general diet group (12.95\%). Notably, genera generally considered to be components of functional flora in a healthy GI tract (e.g. Lachnoclostridium, Dorea, Ruminoccus, Faecalibacterium, Roseburia, and Coprococcus) were significantly more abundant in the general diet group, whereas symbiotic pathogenic bacteria (e.g. Collinsella, Alistipes, and Eggerthella) were more abundant in the gut flora of children in the liquid diet group (all $p<0.05$ ). These results suggest that the different dietary structures of the two groups led to significant differences in gut flora (Table 4). 
As shown in Figure 2, PCA showed a significant difference between the structures of intestinal flora in the liquid diet group compared to that in the general diet group $(p=0.002)$.

\section{Discussion}

In the present study, we found distinctively different gut microbial populations between patients with CP comorbid with epilepsy who consume a diet rich in plant-based solid foods and those fed a liquid diet, with the latter group exhibiting more GI dysfunction symptoms. Prevotella species were predominant in the general diet group, whereas Bifidobacterium species were predominant in the liquid diet group. The gut flora of children in the general diet group contained more butyric acid-producing flora, whereas the gut flora of children in liquid diet group were rich in symbiotic pathogenic bacteria.

\section{The connection among dietary, intestinal flora and constipation}

A multitude of factors can influence the composition and evolution of infant intestinal flora, including gestational age at birth, mode of delivery, mode of feeding, prenatal diet, prenatal use of antibiotics, and living environment. By the age of 3 years old, children should have a mature and stable gut flora, and diet composition becomes the most important factor in determining gut flora. Diet affects the constitution and metabolism of GI microbiota, and nutrient intake is important to the maintenance of gut flora [4]. Compared to European children who were fed with a higher fat diet, African children eating a fiber-rich diet have been reported to have higher level of the Bacteroidetes, especially Prevotella, and Xylanibacter genera, which are related to cellulose and xylan hydrolysis, with relatively low levels of Firmicutes, as well as more short-chain fatty acids (SCFAs) in their GI tracts [5]. High-fat diets have been linked to elevated levels of potentially pathogenic bacteria, especially Fusobacterium species [6]. 
Dietary interventions have been shown to improve the composition and functional status of gut flora [7], which may play an important role in the occurrence and development of constipation [8, 9]. A gut flora imbalance may lead to an insufficiency of probiotics, which impairs digestion and absorption functions, resulting in the accumulation of oligosaccharides in the intestines $[10,11,12]$. It can also disrupt normal intestinal flora composition and metabolite levels — such as by reducing SCFAs, stimulating neurotransmission (e.g. serotonergic transmission), and elevating of neurotoxic metabolites (e.g. lactic acid and ammonia). These changes alter related signaling pathways, leading to dysfunction of the gut-brain axis and impaired intestinal motility, which can cause constipation[10, 11, 12]. Park et al. postulated that colonal motor nerve dysfunction may be a major cause of constipation based on the finding that children with $\mathrm{CP}$ often show delayed emptying of the proximal colon and rectal sigmoid colon [13]. Altogether, this convergence of findings suggests that dietary structure is closely related to intestinal flora, and that insufficient fiber intake, reduced intestinal flora metabolites (e.g. SCFAs), and elevated levels of neurotransmitters and toxic metabolites may lead to constipation.

\section{The influence of dietary fiber and SCFAs on the body}

Dietary fiber is an indigestible form of carbohydrate that acts as a substrate for gut microbial fermentation. A carbohydrate-based diet has been associated with a Prevotella-dominant enterotype, with a higher fiber intake increasing the abundance of Prevotella in the gut $[14,15]$. Dietary fiber promotes the secretion of intestinal mucus, reduces oxygen levels in the gut, and helps to maintain normal physiological intestinal functions. Thus, long-term fiber insufficiency can change the intestinal flora structure, leading to a thinning of the mucus layer, which increases susceptibility to pathogenic bacterial infections and chronic inflammatory diseases. SCFA metabolites can promote differentiation of regulatory $\mathrm{T}$ cells and induce acetylation of histone-H3 in a G-protein-coupled receptor 43-dependent manner, while a high-fat diet has been associated with delayed maturation of the thymus, 
reduced numbers of thymocytes, and apoptosis of developing $\mathrm{T}$ cells; the health-promoting characteristics of dietary fiber appear to depend on microbial metabolite (e.g. SCFA), regulation of physiological changes (such as lowering $\mathrm{pH}$ ) and protection of the mucosal barrier [16,17]. Gut microbe-produced SCFAs (especially butyrate), which are highly concentrated in the cecum and proximal colon, serve as an energy source for colon cells. The major gut SCFAs (acetate, propionate and butyrate) influence the liver and peripheral tissues when they are transported into peripheral circulation through the portal vein. In this way, microbial products play a key role in regulating metabolism, immune function, and cell proliferation.

SCFAs are produced in the colon mainly through fermentation of dietary fiber, digestion-resistant starch, oligosaccharides, and non-digestible sugars by beneficial genera of bacteria, such as Lactobacillus and Bifidobacteria. The physiological effects of SCFAs include: 1) providing energy and regulating electrolytes; 2) protecting the intestinal mucosal barrier (by mechanical and chemical strengthening); 3) influencing $\mathrm{pH}$ and inhibiting the growth of pathogenic bacteria and of conditional pathogens, such as Escherichia coli and Shigella; 4) intestinal immune regulation to resist pathogens; 5) an anti-tumor effect; 6) regulation of intestinal motility; and 7) promotion of intestinal mucosa growth [18-20]. Intake of foods high in carbohydrates can provide the gut with dietary fiber, and thus support the production of SCFAs. Both dietary fiber and SCFAs can help stabilize intestinal flora, thereby strengthening gut barrier protection and supporting immune system development.

\section{Intestinal flora in children in the general diet group}

Prevotella, Roseburia, Lactobacillus, and Faecalibacterium species were found to be significantly higher in children in the general diet group than in the liquid diet group. Prevotella, which represented the most divergent genus between the two groups, can degrade broad-spectrum plant polysaccharides and support carbohydrate-rich food digestion. Besides, it can contribute to the biosynthesis of vitamin 
B1 and support the metabolism of fish oil, both of which are beneficial for brain development $[5,14,21$, 22, 23]. The relatively low incidence of GI dysfunction among children in our general diet reference group, who were able to eat normally and participate in group activities, could be related to their fiber-supplying, carbohydrate-based diet which lead to a high abundance of protective functional bacteria for intestinal barrier such as Prevotella, Lactobacillus, and Faecalis in the intestinal flora. Roseburia species, which accounted for the $10^{\text {th }}$ most prevalent genus in the general diet group, can break down a variety of carbohydrates into SCFAs, including beneficial butyrate, while inhibiting secretion of the proinflammatory cytokine interleukin-17 and promoting the differentiation and maturation of mucosal regulatory $\mathrm{T}$ cells, thereby having an anti-inflammaotry influence on the colon [24,25]. Faecalibacterium can also increase butyrate and exert an anti-inflammatory effect [26]. Butyrate, which is absorbed and used by intestinal epithelial cells, can regulate the growth and development of epithelial cells and act as an energy source. In this way, butyrate can lessen inflammatory colitis and Crohn's disease, and also inhibit proliferation of colon cancer cells [27]. Lactobacillus species are beneficial to host health; they ferment sugar, produce lactic acid, and regulate immune function [28]. As the main energy source for intestinal epithelial cells, butyrate supports the mucosal barrier of the colon and promotes the production of antibacterial peptides. Butyrate can also promote the repair of injured gastric mucosa and provide protection from ulcers by increasing cell proliferation [29]. Our finding of enriched butyrate-producing flora in the intestinal flora of children in the general diet group supports the notion that the low incidence of GI disorders in these children may be consequent to gut flora mediated protection of the intestinal mucosal barrier and maturation of immune function.

\section{Intestinal flora in children from liquid diet group}


Bifidobacterium, Collinsella, Alistipes, and Eggerthella species were found to be significantly higher in the GI tracts of children in the liquid diet group than in those of children in the general diet group, while bacteria related to the digestion of dietary fiber and carbohydrates, such as Prevotella and Lactobacillus species, were markedly decreased. Bifidobacterium is a functional genus of flora found in high abundance in the intestines of breast-fed infants, and its abundance decreases as whole foods are introduced. Thus, intestinal enrichment of Bifidobacterium in childhood reflects an immaturity of intestinal flora [30]. Children in liquid diet group with milk-based liquid diet for a long-term might result in Bifidobacteria mainly in adolescent and infant types, and a lack of Bifidobacterium breve. As a result, the concomitant lack of butyrate-producing flora may compromise immune function and intestinal mucosal integrity. An elevated abundance of Collinsella species has been associated with low dietary fiber intake [31]. Conversely, increasing Collinsella bacteria can reduce the expression of tight junction proteins in intestinal cells, enabling intestinal leakage and, consequently, symptoms of metabolic endotoxemia [32, 33]. An abundance of Alistipes has been related to abdominal pain frequency in patients with irritable bowel syndrome and thus has been speculated to cause intestinal inflammation [34]. Eggerthella lenta is an important human pathogen associated with severe gastrointestinal disorders [35].

Low GI levels of SCFA in the children in our liquid diet group can be attributed to their limited intake of dietary fiber and carbohydrates. Such a SCFA deficiency can lead to a compromised state of intestinal barrier protection, which is normally associated an adequate presence of Clostridium and Lactobacillus species. Conversely, an elevated presence of potentially pathogenic bacteria, including Collinsella, Alistipes, and Eggerthella, can lead to chronic inflammation in the intestinal tract and a weak immune system function in the GI tract. As a result, proliferation of potentially pathogenic species, such as Klebsiella pneumoniae, Pseudomonas aeruginosa, and Escherichia coli, can lead to endogenous infection of the intestinal tract. Repeated antibiotic treatment to reduce the presence of 
these pathogenic microbes can, eventually, lead to drug-resistant strains and a consequent transition from an endogenous infection to an exogenous infection, resulting in recurrent GI infections requiring repeated hospitalizations [36]. Moreover, serious intestinal flora imbalances which caused by the factors above can aggravate GI dysfunction symptoms, which further limits the absorption of essential nutrients, thereby forming a vicious cycle. Our findings showing deficiencies in butyrate- and lactate-producing gut bacteria in the liquid diet group suggest that these children have an immature complement of symbiotic pathogenic bacteria that does not support maintenance of the protective intestinal barrier or immune function, and these conditions may underlie their increased prevalence of GI dysfunction.

\section{Research summary and further research ideas}

Children with CP and epilepsy who eat a diet rich in plant-based solid foods were found to have abundant levels of beneficial bacteria, including Roseburia, Lactobacillus, and Faecalibacterium, in their intestinal flora, which is conducive to the digestion of dietary fiber and enables the production of SCFAs, which protect the intestinal mucosa while promoting immune development and maturation. Relatively decreased levels of protective functional bacteria with elevated levels of pathogenic bacteria in children fed a (semi)liquid diet may impede development of immune function. This underdeveloped immune function can lead to chronic gut inflammation and GI dysfunction. The most common GI dysfunction symptom, obstipation, can reduce quality of life severely. Although the Bifidobacterium genus was abundant in this group of children, the functionally important species Bifidobacterium breve was lacking.

In the present study, our gene sequence analysis was limited to the 16SrRNA gene. Thus there is a need for a macro factor analysis in future research. In addition, it will be important to examine the effects of dietary fiber and Lactobacillus and Tyrobacter intervention on changes in gut flora and GI 
dysfunction symptoms in children with $\mathrm{CP}$ and epilepsy who eat a liquid or semi-liquid diet. Additionally, because our study population was limited to children cared for at the Longgang District Social Welfare Center, it is not known whether similar diet-related disparities in gut flora and GI dysfunction would be found among children with CP and epilepsy who are raised in their family homes. Thus, larger-sample, multi-center and clinical intervention studies are needed to determine the generalizability of the findings obtained here.

\title{
Conclusions
}

The present study explored the effect of different dietary structure which including the liquid diet and the general diet on gastrointestinal dysfunction in children with cerebral palsy and epilepsy. And it demonstrated that there was a huge difference on gut microbial populations between the two groups. The intestinal flora of children with CP and epilepsy consuming a liquid diet had elevated levels of symbiotic pathogens and diminished intestinal barrier protection bacteria, while for children in the general diet group the intestinal flora contained more butyric acid-producing flora which was also common in healthy people. Even though there were some limitations in the study, it inspires clinical staffs who are working on improving the quality of life among the children with cerebral palsy and epilepsy and provides theoretical basis for the following intervention.

\author{
Abbreviations \\ CP: Cerebral palsy \\ FDR: false discovery rates \\ GI: Gastrointestinal \\ PCA: Principle component analysis \\ SCFA: short-chain fatty acids \\ SD: standard deviations
}




\section{Declarations}

\section{Ethics Approval and Consent to Participate}

This study was approved by the Ethics Committee of the Longgang District Maternity \& Child Healthcare Hospital in Shenzhen, China (registration number LGFY2017005). Written informed consent was obtained through Longgang District Social Welfare Center which supports scientific research and investigation of the children in its care.

\section{Consent for publication}

Not applicable

\section{Availability of data and materials}

The dataset generated for this study can be viewed in the NCBI sequence archive under biological project number PRJNA530084.

\section{Competing interests}

The authors declare that they have no competing interests.

\section{Funding}

This work has received strong support from the Longgang District Science and Technology Innovation Bureau (no. p020180720388406844161) and the Shenzhen Science and Technology Project (no. JCYJ20170816170527583).

\section{Authors' contributions}

Huang Congfu, Zhang Anquan, Yang Zhenyu, and Ge Lan managed the project. Peng Yuanping, Huang Shuyuan, Wang Peiqin, and Li Xiuyun took fecal samples and collected information. Huang Congfu, Zhang Anquan, and Yang Zhenyu prepared DNA. Yang Zhenyu and Dai Wenkui were 
responsible for the bioinformatics analysis. Huang Congfu, Ge Lan, and Li Xiuyun analyzed and interpreted the results and wrote the manuscript. Yang Zhenyu, Zhang Anquan, and Wu Genfeng optimized the graphics and statistical analyses. Dai Wenkui directed the project design and revised the article. Lyu Yansi and Wang Linlin helped with the writing of the manuscript. All of the authors reviewed the manuscript before submission.

\section{Acknowledgements}

We thank all of the study participants and their parents for their participation. We are grateful to the physicians and nurses at the Longgang District Maternity \& Child Healthcare Hospital in China and the professionals at the Longgang district Social Welfare Center who assisted the research team with clinical examinations and stool sample collection. 


\section{References}

[1] Rehabilitation Group, Pediatrics Branch, Chinese Medical Association, Neurology Group, Pediatrics Branch, Chinese Medical Association. Expert consensus on diagnosis and treatment of epilepsy in patients with comorbid cerebral palsy. Chinese Journal of Clinical Pediatrics, 2017, 32 (16): 1222- 1226. Article in Chinese

\section{http://med.wanfangdata.com.cn/Paper/Detail?dbid=WF_QK\&id=syeklczz201716006}

[2] Quitadamo P, Thapar N, Staiano A, Borrelli O. Gastrointestinal and nutritional problems in neurologically impaired children. Eur J Paediatr Neurol, 2016, 20(6): 810-815.

[3] Huang CF, Peng YP, Li YH, Dai WK, Xiong SH, Wu GF, Li XY, Tang HZ. Changes of intestinal flora in children with cerebral palsy and epilepsy. Chinese Journal of Microecology, 2019, 31 (4): 402-406. Article in Chinese

http://med.wanfangdata.com.cn/Paper/Detail?dbid=WF_QK\&id=PeriodicalPaper_zgwstxzz201904006

[4] Scott KP, Gratz SW, Sheridan PO, Flint HJ, Duncan SH. The influence of diet on the gut microbiota. Pharmacological Research, 2013, 69 (1): 52-60.

[5] Filippo CD, Cavalieri D, Paola, MD, Ramazzotti M, Poullet JB, Massart S, Collini S, Piearaccini G, Lionetti P. Impact of diet in shaping gut microbiota revealed by a comparative study in children from Europe and rural Africa. Proc Natl Acad Sci, 2010, 107: 14691 -14696.

[6] Xia Y, Zhu QC, Wang Y, Peng JY, Qian HX. Correlation between changes in intestinal flora caused by high-fat diets and the occurrence of colorectal cancer. Chinese General Practice, 2016,19 (20): 2473-2480 . Article in Chinese

\section{http://med.wanfangdata.com.cn/Paper/Detail/PeriodicalPaper zgqkyx201620025}

[7] Zhao LP, Zhang CH, Fei N, Zhang X, Xiao SM. Research progress on nutritional interventions for metabolic diseases targeting intestinal flora. Chinese Journal of Food Science, 2014,14 (1): 1-5. Article in Chinese 
[8] Dimidi E, Christodoulides S, Scott SM, Whelan K. Mechanisms of Action of Probiotics and the Gastrointestinal Microbiota on Gut Motility and Constipation. Adv Nutr, 2017; 8: 484-94.

[9] Huang RX, Hu JA. Positive Effect of Probiotics on Constipation in Children: A Systematic Review and Meta-Analysis of Six Randomized Controlled Trials. Front. Cell. Infect. Microbiol., 2017, 7: 153.

[10] $\mathrm{Yu} \mathrm{Y.} \mathrm{Correlation} \mathrm{of} \mathrm{senile} \mathrm{constipation} \mathrm{with} \mathrm{intestinal} \mathrm{flora} \mathrm{imbalance} \mathrm{and} \mathrm{pharmacological}$ intervention. Journal of Gastroenterology and Hepatology, 2010,19 (12): 1133-1135. Article in Chinese

http://med.wanfangdata.com.cn/Paper/Detail?dbid=WF_QK\&id=PeriodicalPaper_wcbxhgbxzz201012 022

[11] Shen XX, Yu T, Lin L. How the gastrointestinal microecology affects chronic constipation and guides clinical treatment.Gastroenterology, 2017, 22 (5): 308-311. Article in Chinese

http://www.cnki.com.cn/Article/CJFDTOTAL-WIEC201705011.htm

[12] Galland L. The gut microbiome and the brain. J Med Food, 2014, 17 (12): 1261-1272.

[13] Park ES, Park CI, ChoSR, Na SI, Cho YS. Colonic transit time and constipation in children with spastic cerebral palsy. Arch Phys Med Rehabil, 2004, 85 (3): 453-456.

[14] Wu GD, Chen J, Hoffmann C, Bittinger K, Chen YY, Keilbaugh SA, Bewtra M, Knights D, Walters WA, Knight R, Sinha R, Gilroy E, Gupta K, Baldassano R, Nessel L, Li HZ, Bushman FD, Lewis JD. Linking long-term dietary patterns with gut microbial enterotypes. Science, 2011, 334 (6052): 105-108.

[15] Kovatcheva-Datchary P,Nilsson A, AkramiR, Lee YS, Vadder FD, Arora T, Hallen A, Martens E, Björck I, Bäckhed F. Dietary Fiber-Induced Improvement in Glucose Metabolism Is Associated with Increased Abundance of Prevotella. Cell Metabolism, 2015, 22 (6): 971-982.

[16] Makki K, Deehan EC, Walter J, Bäckhed F. The Impact of Dietary Fiber on Gut Microbiota in 
Host Health and Disease. Cell Host \& Microbe, 2018, 13 (23): 705-715.

[17] Sun CB, Liu ZJ, Liu F, Du P, Hou JC. Effects of food ingredients on the structure of intestinal flora. Food Research and Development, 2018, 39 (9): 178-182. Article in Chinese

http://www.wanfangdata.com.cn/details/detail.do?_type=perio\&id=spyjykf201809035

[18] Salonen A, VOS WM D. Impact of Diet on Human Intestinal Microbiota and Health. Annual Review of Food Science \& Technology, 2014, 5 (1): 239-262.

[19] Kabat AM, Srinivasan N, Maloy KJ. Modulation of immune development and function by intestinal microbiota. Trends Immunol, 2014, 35 (11): 507-517.

[20] Meng DL, Liang LX, Song HY. Physiological role of short-chain fatty acids in the intestine. Chinese Journal of New Medicine, 2018, 11 (2): 198-202. Article in Chinese

\section{http://med.wanfangdata.com.cn/Paper/Detail?id=PeriodicalPaper_zgyxwz-lnyxfc201802030}

[21]] Arumugam M, Raes J, Pelletier E, Le Paslier D, Yamada T. Enterotypes of the human gut microbiome. Nature, 2011, 473: 174-180.

[22] Huws SA, Kim EJ, Lee MRF, Scott MB, Tweed JKS, Pinloche E, Wallace RJ, Scollan ND. As yet uncultured bacteria phylogenetically classified as Prevotella, Lachnospiraceae incertae sedis and unclassified Bacteroidales, Clostridiales and Ruminococcaceae may play apredominant role in ruminal biohydrogenation Environmental. , 2011, 13: 1500-1512.

[23] Tremlett H, Bauer KC, Appel-Cresswell S, Cresswell SA, Finlay BB, Waubant E. The gut microbiome in human neurological disease: A review. Ann Neurol, 2017, 81 (3): 369-382.

[24] Travis AJ, Kelly D, Flint HJ, Aminov RI. Complete Genome Sequence of the Human Gut Symbiont Roseburia hominis. Genome Announc, 2015; 3 (6): e01286-15.

[25] Zhu CX, Song KR, Shen ZH, Quan YS, Tan B, Luo WW, Wu S, Tang K, Yang ZY, Wang XY . Roseburia intestinalis inhibits interleukin-17 excretion and promotes regulatory $\mathrm{T}$ cells differentiation in colitis. Molecular Medicine Reports, 2018; 17: 7567-7574. 
[26] Louis P,Flint HJ. Diversity,metabolism and microbial ecology of butyrate-producing bacteria from the human large intestine. FEMS Microbiol,2009,294(1):1-8.

[27] Remely M, Aumueller E, Merold C, Dworzak S, Hippe B, Zanner J, Pointner A, Brath H, Haslberger AG. Effects of short chain fatty acid producing bacteria on epigenetic regulation of FFAR3 in type 2 diabetes and obesity. Gene, 2014,537 (1): 85-92 .

[28] Zhang ZW, Lv JL, Pan L, Zhang YG. Roles and applications of probiotic Lactobacillus strains. Applied Microbiology and Biotechnology,2018,102:8135-8143.

[29] Wilson AJ, Gibson PR. Short-chain fatty acids promote the migration of colonic epithelial cells in vitro. Gastroenterology, 1997, 113 (2): 487-496.

[30] Zhong HZ, Penders J, Shi J, Ren Huahui, Cai KY, Fang C, Ding QX, Thijs C,. Impact of early events and lifestyle on the gut microbiota and metabolic phenotypes in young school-age children. Microbiome, 2019; 7: 2.

[31] Gomez-Arango LF, Barrett HL, Wilkinson SA, Callaway LK, Mclntyre HD, Morrison M, Nitert MD. Low dietary fiber intake increases Collinsella abundance in the gut microbiota of overweight and obese pregnant women. Gut Microbes, 2018; 9 (3): 189- 201.

[32] Chen J, Wright K, Davis JM, Jeraldo P, Marietta EV, Murray J, Nelson H, Matteson EL, Taneja V. An expansion of rare lineage intestinal microbes characterizes rheumatoid arthritis. Genome Med 2016; 8: 43 .

[33] Cani PD, Possemiers S, Van de Wiele T, Guiot Y, Everard A, Rottier O, Geurts L, Naslain D, Neyrinck A, Lambert DM, Mucciolo GG, Delzenne NM. Changes in gut microbiota control in fl ammation in obese mice through a mechanism involving GLP-2-driven improvement of gut permeability.Gut. 2009; 58: 1091-103.

[34] Saulnier DM, Riehle K, Mistretta TA, Diaz MA, Mandal D, Raza S, Weidler EM, Qin X, Coarfa C, Milosavljevic A, Petrosino JF, Highlander S, Gibbs R, Lynch SV, Shulman RJ, Versalovic J. 
Gastrointestinal microbiome signatures of pediatric patients with irritable bowel syndrome. Gastroenterology, 2011, 141 (5): 1782-1791.

[35] Gardiner BJ, Tai AY, Kotsanas D, Francis MJ, Roberts SA, Ballard SA, Junckerstorff RK, Korman TM. Clinical and Microbiological Characteristics of Eggerthella lenta Bacteremia. Journal of Clinical Microbiology, 2015, 53 (2): 626-635.

[36] Wu QB, Zheng YJ, Huang YK. Intestinal flora of children-basic and clinical. 2nd edition, Science Press, 2019: 1-403. .Book in Chinese 


\section{Figure legends}

Fig 1. Histogram of relative abundance of bacterial phyla based on bacterial structure analysis. Phylum percentages are reported in Table 2. Only Bacteroides and Actinobacteria percentages differed significantly (see Results text) between the liquid diet $(\mathrm{N}=23)$ and general $\operatorname{diet}(\mathrm{N}=17)$ groups.

Fig 2. PCA comparison between study groups of intestinal flora. Each specimen (one per patient) is represented by a data point (red, general diet; blue, liquid diet). PCA revealed a significant inter-group structural difference $(p=0.002)$. 


\section{Tables}

Table 1 Inter-group comparison of the prevalence $(N)$ of $\mathrm{GI}$ dysfunction symptoms in pediatric patients with CP and epilepsy.

\begin{tabular}{lccc}
\hline Symptom & $\begin{array}{c}\text { General diet group } \\
\mathbf{N}=\mathbf{1 7}\end{array}$ & $\begin{array}{c}\text { Liquid diet group } \\
\mathbf{N}=\mathbf{2 3}\end{array}$ & $\boldsymbol{P}$ \\
\hline Obstipation & 1 & 23 & $<0.001$ \\
Bloating & 1 & 8 & $<0.05$ \\
Diarrhea & 2 & 0 & $>0.05$ \\
Vomiting & 2 & 10 & $<0.05$ \\
Gl bleeding & 0 & 6 & $<0.05$ \\
\hline
\end{tabular}

Table 2 Top five most prevalent bacterial phyla in each group.

\begin{tabular}{lcllc}
\hline \multicolumn{2}{c}{ General diet group } & & \multicolumn{2}{c}{ Liquid diet group } \\
\cline { 1 - 2 } \cline { 5 - 5 } Top 5 phyla & Mean \pm SD \% & & Top 5 phyla & Mean \pm SD \% \\
\hline Bacteroidetes & $41.83 \pm 27.12$ & & Firmicutes & $35.25 \pm 17.71$ \\
Firmicutes & $34.28 \pm 15.00$ & & Actinobacteria & $30.99 \pm 18.42$ \\
Actinobacteria & $16.68 \pm 20.69$ & & Bacteroidetes & $25.45 \pm 17.92$ \\
Proteobacteria & $4.96 \pm 3.94$ & & Proteobacteria & $4.96 \pm 4.21$ \\
Fusobacteria & $0.94 \pm 1.43$ & & Verrucomicrobia & $2.07 \pm 2.49$ \\
\hline
\end{tabular}


Table 3 Top 15 genera of gut microbes in terms of representation by group.

\begin{tabular}{lcllc}
\hline \multicolumn{2}{c}{ General diet group } & & \multicolumn{2}{c}{ Liquid diet group } \\
\cline { 1 - 2 } \cline { 5 - 5 } Top 15 genera & Mean \pm SD (\%) & & Top 15 genera & $\begin{array}{c}\text { Mean } \pm \text { SD } \\
\text { (\%) }\end{array}$ \\
\hline Prevotella & $25.85 \pm 26.11$ & & Bifidobacterium & $24.93 \pm 15.60$ \\
Bifidobacterium & $12.95 \pm 17.04$ & & Bacteroides & $12.13 \pm 11.31$ \\
Bacteroides & $8.45 \pm 8.91$ & & Enterococcus & $6.15 \pm 16.59$ \\
Parabacteroides & $3.92 \pm 7.47$ & & Parabacteroides & $5.13 \pm 4.92$ \\
Streptococcus & $2.79 \pm 3.24$ & & Collinsella & $4.51 \pm 3.96$ \\
Faecalibacterium & $2.32 \pm 2.19$ & & Prevotella & $3.61 \pm 7.38$ \\
Collinsella & $2.15 \pm 4.05$ & & Streptococcus & $2.71 \pm 3.65$ \\
Sutterella & $1.68 \pm 1.94$ & & Akkermansia & $2.02 \pm 2.51$ \\
Acidaminococcus & $1.35 \pm 3.95$ & & Megasphaera & $1.79 \pm 3.10$ \\
Roseburia & $1.35 \pm 1.78$ & & Blautia & $1.48 \pm 2.79$ \\
Megasphaera & $1.33 \pm 3.87$ & & Alistipes & $1.45 \pm 2.29$ \\
Alloprevotella & $1.32 \pm 1.82$ & & Eubacterium & $1.40 \pm 4.45$ \\
Enterococcus & $1.13 \pm 2.78$ & & Faecalibacterium & $1.16 \pm 2.24$ \\
Catenibacterium & $1.02 \pm 1.71$ & & Desulfovibrio & $0.87 \pm 1.97$ \\
Megamonas & $1.02 \pm 3.13$ & & Sutterella & $1.68 \pm 1.94$ \\
\hline
\end{tabular}


Table 4 Intestinal flora genera with significantly differing levels between the general diet and liquid diet groups.

\begin{tabular}{|c|c|c|c|}
\hline \multirow[b]{2}{*}{ Bacteria（Genus） } & \multicolumn{2}{|c|}{ Mean Value } & \multirow[b]{2}{*}{ P Value } \\
\hline & $\begin{array}{c}\text { The } \\
\text { General } \\
\text { Diet } \\
\text { Group } \\
(\%)\end{array}$ & $\begin{array}{c}\text { The } \\
\text { Liquid } \\
\text { Diet } \\
\text { Group } \\
(\%)\end{array}$ & \\
\hline Eggerthella & 0.007 & 0.065 & 0.001 \\
\hline Prevotella & 25.849 & 3.612 & 0.001 \\
\hline Lachnoclostridium & 0.236 & 0.763 & 0.001 \\
\hline Dorea & 0.160 & 0.053 & 0.001 \\
\hline Coprococcus & 0.258 & 0.053 & 0.002 \\
\hline Ruminococcus & 0.388 & 0.232 & 0.002 \\
\hline Faecalibacterium & 2.324 & 1.156 & 0.002 \\
\hline Lactobacillus & 0.582 & 0.060 & 0.003 \\
\hline Roseburia & 1.347 & 0.093 & 0.004 \\
\hline Turicibacter & 0.008 & 0.005 & 0.006 \\
\hline Collinsella & 2.153 & 4.510 & 0.007 \\
\hline Corynebacterium & 0.150 & 0.089 & 0.011 \\
\hline Alistipes & 0.351 & 1.453 & 0.013 \\
\hline Bifidobacterium & 12.947 & 24.929 & 0.013 \\
\hline Anaerococcus & 0.001 & 0.013 & 0.014 \\
\hline
\end{tabular}

Difference screening conditions: average relative abundance $>0.1 \%, p<0.005$, and FDR $<$ 0.05 . 


\section{Supplementary material}

Supplementary materials associated with this article are available online at:

https://www.frontiersin.org/articles/10.3389/fped.2019.00394/full\#

Supplementary Document 1 | Background information of registered subjects.

Supplementary Document $2 \mid$ Phylum and genus distributions of genetically modified organisms in all study subjects.

Supplementary Document $3 \mid$ Functional distribution of gut microbiota of study subjects according to the KEGG database. 
Fig. 1

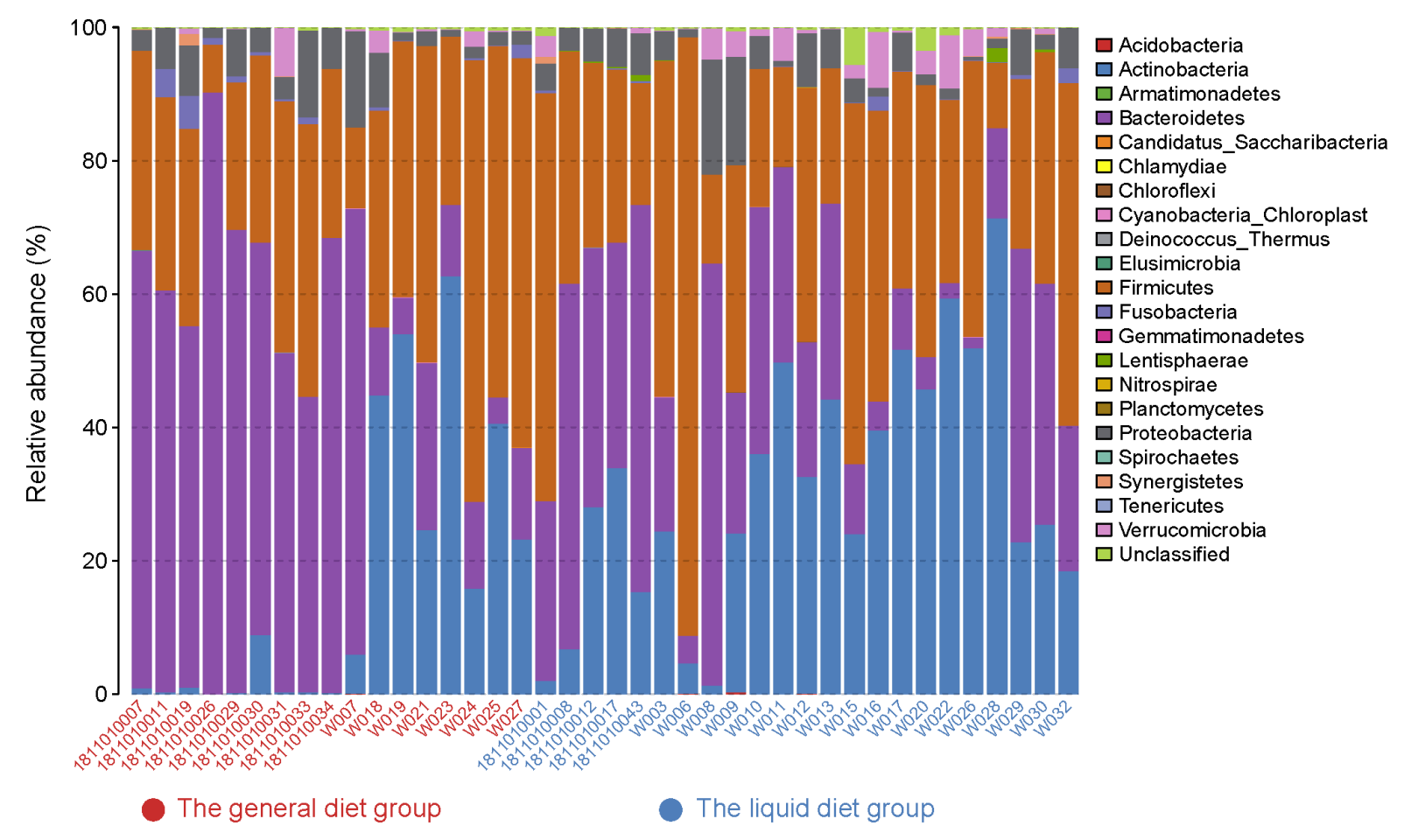


Fig 2.

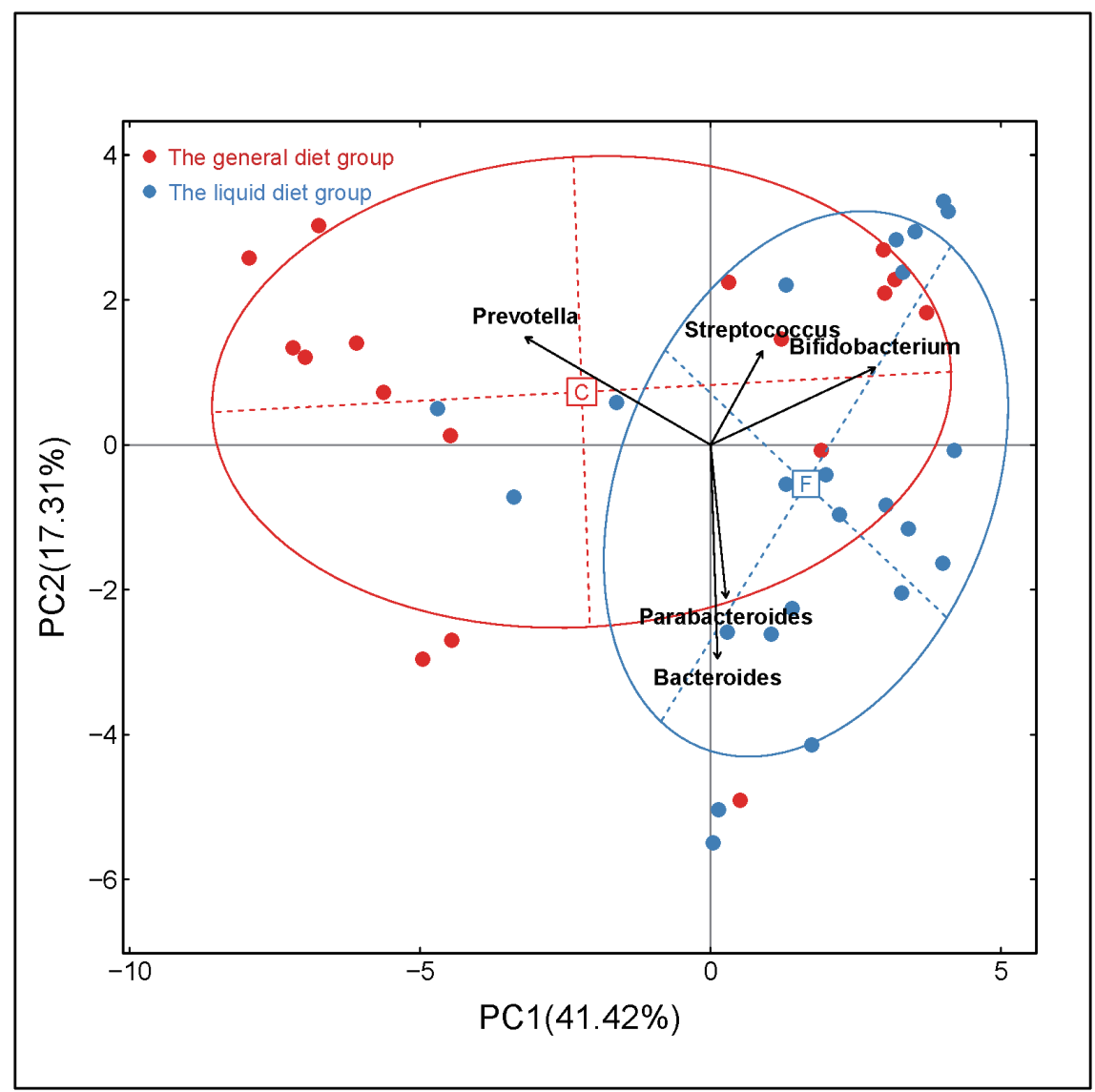




\section{Figures}

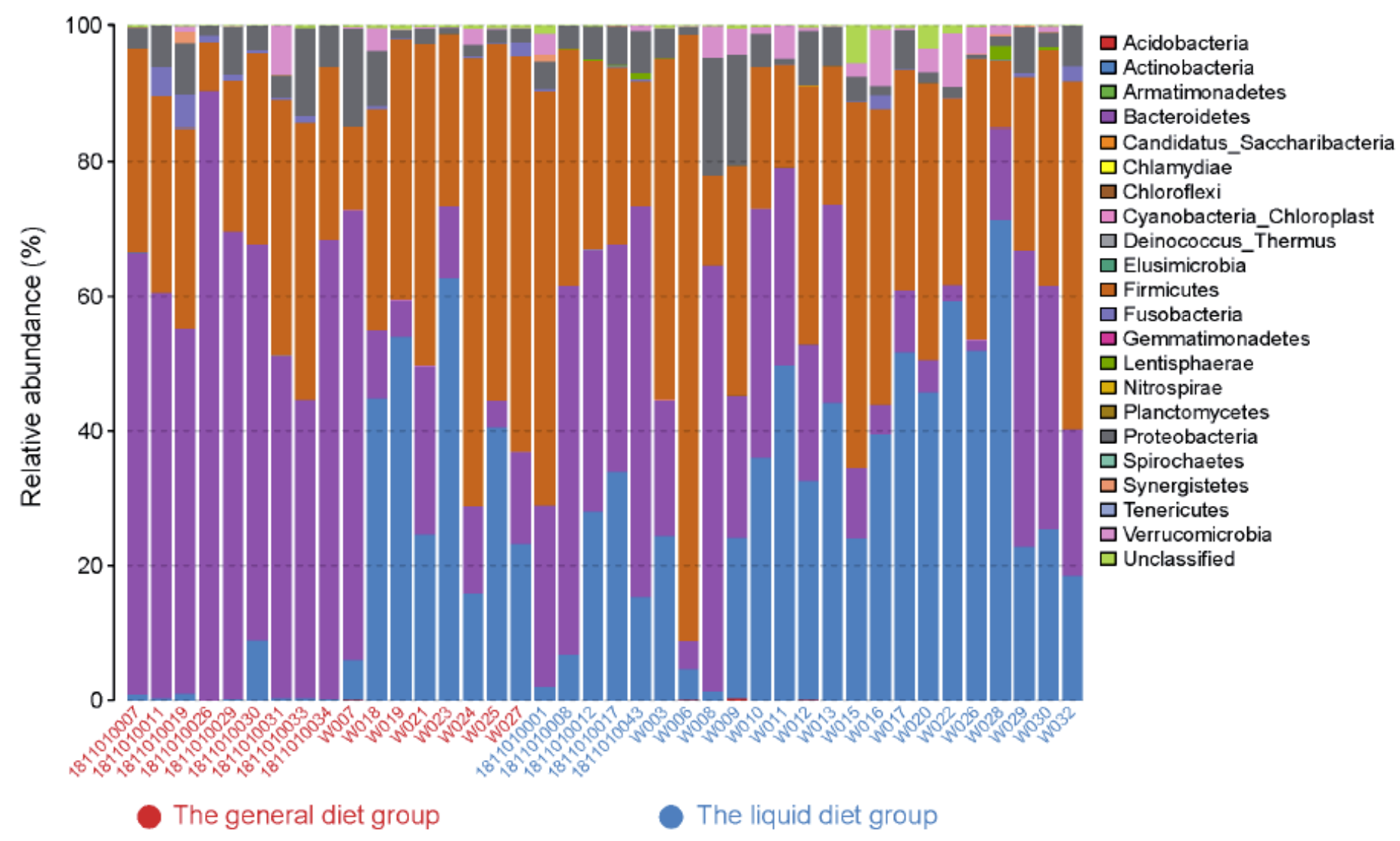

\section{Figure 1}

Histogram of relative abundance of bacterial phyla based on bacterial structure analysis. Phylum percentages are reported in Table 2. Only Bacteroides and Actinobacteria percentages differed significantly (see Results text) between the liquid diet $(N=23)$ and general diet $(N=17)$ groups. 


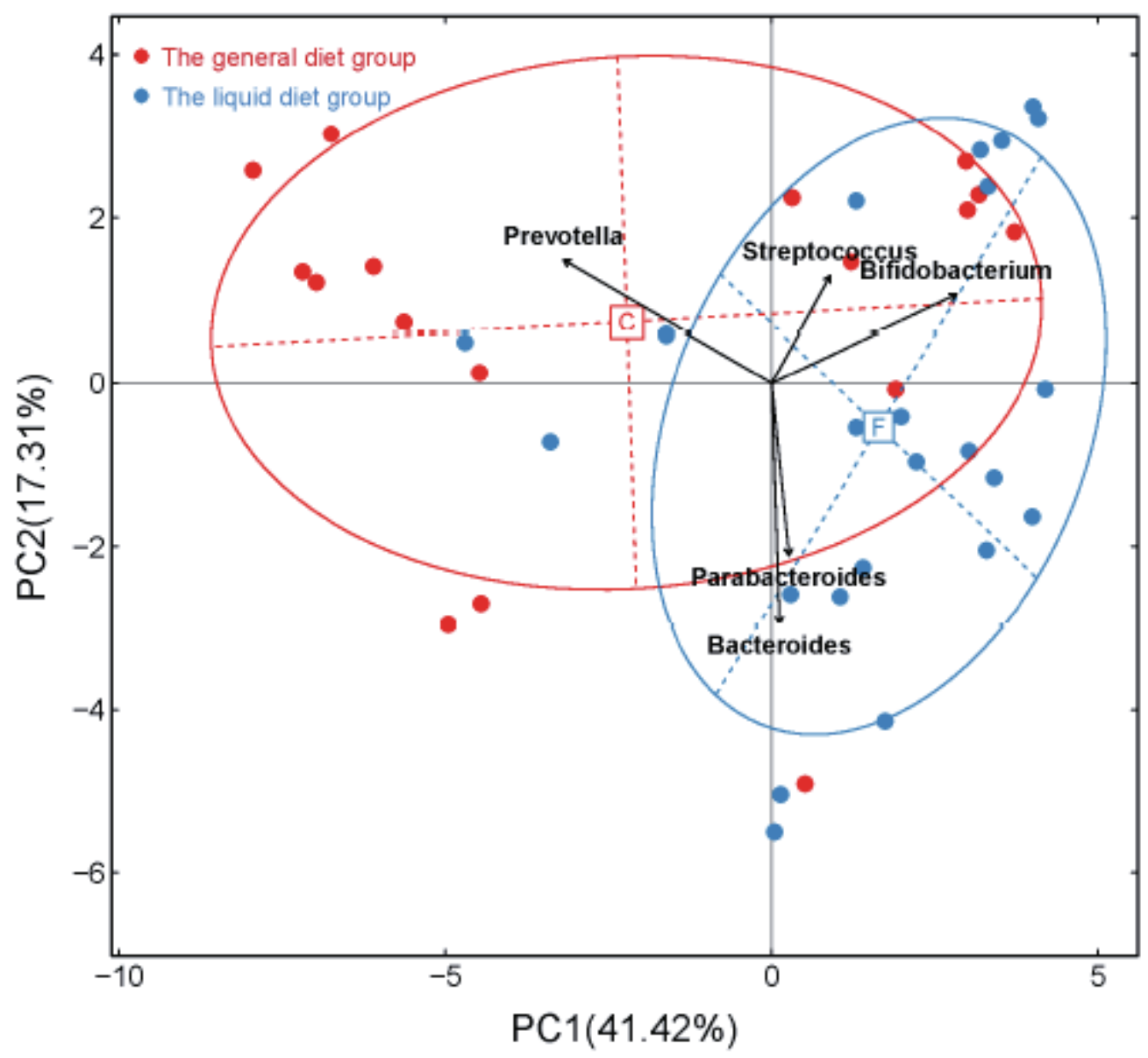

Figure 2

PCA comparison between study groups of intestinal flora. Each specimen (one per patient) is represented by a data point (red, general diet; blue, liquid diet). PCA revealed a significant inter-group structural difference $(p=0.002)$.

\section{Supplementary Files}

This is a list of supplementary files associated with this preprint. Click to download.

- Supplementarymaterial.pdf 